Discrete Comput Geom 29:279-303 (2003)

DOI: $10.1007 / \mathrm{s} 00454-002-0759-2$

\title{
Reconstruction of Convex Bodies from Brightness Functions*
}

\author{
R. J. Gardner ${ }^{1}$ and Peyman Milanfar ${ }^{2}$ \\ ${ }^{1}$ Department of Mathematics, Western Washington University, \\ Bellingham, WA 98225-9063, USA \\ gardner@ baker.math.wwu.edu \\ ${ }^{2}$ Department of Electrical Engineering, University of California, \\ 1156 High Street, Santa Cruz, CA 95064-1077, USA \\ milanfar@ee.ucsc.edu
}

\begin{abstract}
Algorithms are given for reconstructing an approximation to an unknown convex body from finitely many values of its brightness function, the function giving the volumes of its projections onto hyperplanes. One of these algorithms constructs a convex polytope with less than a prescribed number of facets, while the others do not restrict the number of facets. Convergence of the polytopes to the body is proved under certain essential assumptions including origin symmetry of the body. Also described is an oracle-polynomialtime algorithm for reconstructing an approximation to an origin-symmetric rational convex polytope of fixed and full dimension that is only accessible via its brightness function. Some of the algorithms have been implemented, and sample reconstructions are provided.
\end{abstract}

\section{Introduction}

The brightness function of a convex body in $\mathbb{R}^{n}$ gives the $((n-1)$-dimensional) volume of its (orthogonal) projections onto hyperplanes. (See Section 2 for unexplained terms and notation.) Aleksandrov's projection theorem states that any two origin-symmetric convex bodies in $\mathbb{R}^{n}$ that have the same brightness function must be equal; see, for example, Theorem 3.3.6 of [8]. Published in 1937, and utilizing radically new machinery developed at the time by Aleksandrov, this result is quite remarkable since the data is so weak; only the volumes of the projections, and nothing about their shapes, is known. However, Aleksandrov's theorem does not provide a method by which an origin-symmetric convex body can be reconstructed from its brightness function. The main purpose of this paper

\footnotetext{
* This research was supported in part by U.S. National Science Foundation Grants DMS-9802388, DMS0203527, and CCR-9984246.
} 
is to provide algorithms for such a reconstruction, thereby solving a basic problem in geometric tomography (see Problem 3.6 of [8]), the area of mathematics concerning the retrieval of information about a geometric object from data concerning its sections or projections.

Suppose that $n \geq 2,0<r<R$, and $K$ is an unknown origin-symmetric convex body in $\mathbb{R}^{n}$ such that $r B \subset K \subset R B$, where $B$ is the unit ball. Given the values of the brightness function of $K$ in a set $U_{k}=\left\{u_{i}: i=1, \ldots, k\right\}$ of directions such that $U_{k} \cup-U_{k}$ is spaced sufficiently closely in $S^{n-1}$ in terms of $n, r$, and $R$, our algorithms construct an originsymmetric convex polytope $P_{k}$ that has the same brightness function as $K$ in the directions in $U_{k}$. We actually describe two variants, one (Algorithms BrightLSQ and BrightLP) in which an upper bound for the number of facets of $P_{k}$ is not prescribed, and the other (Algorithm BrightNFacets) in which this number is prescribed. Algorithm BrightNFacets might be suitable if it is known a priori that the convex body $K$ is a polytope (in which case an upper bound for the number of its facets is quite likely also known), while Algorithms BrightLSQ or BrightLP should be used if it is not known whether $K$ is a polytope.

In Theorems 6.1 and 6.2 below, we show that if $\left\{ \pm u_{i}\right\}$ is a dense set of directions in $S^{n-1}$, then $P_{k} \rightarrow K$ in the Hausdorff metric as $k \rightarrow \infty$. Moreover, the proofs provide estimates on the rate of convergence as a function of $n, R$, and a measure of the spread of the vectors $u_{i}, i=1, \ldots, k$, in $S^{n-1}$. The extra assumption that $K$ is also a convex polytope with the prescribed number of facets is of course necessary for convergence in Algorithm BrightNFacets. The hypotheses of the denseness of $\left\{ \pm u_{i}\right\}$ and the origin symmetry of $K$ are also necessary, as is explained at the end of Section 6.

The algorithms proceed in two phases. In Phase I the brightness measurements are used to find the surface area measure of the approximating convex polytope $P_{k}$. Since the brightness function of a convex body is, up to a constant, the cosine transform of its surface area measure, Phase I essentially requires the inversion of the cosine transform. In Phase II $P_{k}$ is reconstructed from its surface area measure.

An algorithm for Phase II was first published by Little [23]. As far as we know, three groups of researchers have considered this part of our problem since then, each apparently unaware of the others. Sumbatyan and Troyan [26] provide a method based on solving nonlinear PDEs, for the purpose of reconstructing a cavity from ultrasound. Kaasalainen, Lamberg, Lumme, and Boswell (see [18] and the references given there) treat a problem in astrophysics in which the shape of a rotating atmosphereless object such as an asteroid is to be reconstructed by a method called disk-integrated photometry; these authors employ a considerably more elaborate version of Little's algorithm. Motivated by computer vision, Lemordant et al. [22] also modify Little's algorithm, and it is their approach that seems the most appropriate for our purposes. In Section 3 we present this as Algorithm MinkData, since it does not appear to be well known to the geometry community. We shall also have use for a polynomial-time algorithm due to Gritzmann and Hufnagel [11] for reconstructing, in a fixed dimension, a convex polytope whose surface area measure is approximately equal to a given one.

Phase I has also been considered before, though in a quite different context. In [17] Kiderlen needs to invert the cosine transform in order to estimate the directional distribution of a fiber process from its rose of intersection, a function on the unit sphere 
giving certain averages of intersections of the process with hyperplanes through the origin. One of his algorithms is a linear program based on a result of Campi et al. [6], and this can also be applied to our situation in the case when the number of facets of the reconstructed polytope is left free (see Algorithm BrightLP below). Before we learned of the work of Kiderlen, we developed a least squares approach for this purpose (Algorithm BrightLSQ below). We are grateful to Markus Kiderlen for drawing our attention to Algorithm BrightLP.

In Section 7 below, we present an oracle-polynomial-time algorithm for reconstructing an approximation to an origin-symmetric rational convex polytope of fixed and full dimension that is only accessible via its brightness function. This relies on the polynomialtime algorithm for Phase II in [11] (which, however, has not been implemented) and the linear program approach to Phase I in Algorithm BrightLP.

While we are concerned in this paper only with the solution of a problem in convex geometry/geometric tomography in which the input brightness function values are given exactly, it is important to stress that experiments suggest that our algorithms also work with noisy data. In fact, this is one reason why we provide both Algorithm BrightLSQ and Algorithm BrightLP. For exact data, the linear program (LP) in Algorithm BrightLP should run faster. (At least in regard to Phase I; see the comments in Section 10.) When the data is noisy, however, the constraint (21) may cause solutions to degenerate, even to a single point, a problem that does not occur with the program (LLS) in Algorithm BrightLSQ. The presence of noise is unavoidable in practice, and affects performance and stability of any algorithm. For this reason we prefer not to provide a performance analysis in this paper, but rather to do this in a future paper focusing on noisy data and directed towards the engineering community, where geometrical reconstruction problems of this type are of considerable interest. For example, the simpler problem of reconstruction from noisy support function measurements has already been studied fairly extensively; see [16], [20], and [21].

\section{Definitions, Notation, and Preliminaries}

As usual, $S^{n-1}$ denotes the unit sphere, $B$ the unit ball, $o$ the origin, and $\|\cdot\|$ the norm in Euclidean $n$-space $\mathbb{R}^{n}$. It is assumed throughout that $n \geq 2$. A direction is a unit vector, that is, an element of $S^{n-1}$. If $u$ is a direction, then $u^{\perp}$ is the $(n-1)$-dimensional subspace orthogonal to $u$. If $x, y \in \mathbb{R}^{n}$, then $x \cdot y$ is the inner product of $x$ and $y$ and $[x, y]$ denotes the line segment with endpoints $x$ and $y$.

Let $0<\varepsilon<1$. A set $U \subset S^{n-1}$ is called an $\varepsilon$-net if each point in $S^{n-1}$ is within a distance $\varepsilon$ of some point in $U$.

If $A$ is a set, $\operatorname{dim} A$ is its dimension, that is, the dimension of its affine hull, and $\partial A$ is its boundary. The notation for the usual orthogonal projection of $A$ on a subspace $S$ is $A \mid S$.

We write $V_{k}$ for $k$-dimensional Lebesgue measure in $\mathbb{R}^{n}$, where $k=1, \ldots, n$, and where we identify $V_{k}$ with $k$-dimensional Hausdorff measure. If $K$ is a $k$-dimensional convex body in $\mathbb{R}^{n}$, then $V(K)$ is its volume $V_{k}(K)$. Define $\kappa_{n}=V(B)$. The notation $d z$ will always mean $d V_{k}(z)$ for the appropriate $k=1, \ldots, n$. 
A set is origin symmetric if it is centrally symmetric, with center at the origin.

If $K$ is a compact convex set in $\mathbb{R}^{n}$,

$$
h_{K}(x)=\max \{x \cdot y: y \in K\}
$$

is its support function and

$$
b_{K}(u)=V\left(K \mid u^{\perp}\right),
$$

for $u \in S^{n-1}$, its brightness function.

Let $K$ be a convex body in $\mathbb{R}^{n}$, that is, a compact convex set with nonempty interior. The projection body of $K$ is the origin-symmetric convex body $\Pi K$ defined by

$$
h_{\Pi K}=b_{K} .
$$

An introduction to the theory of projection bodies is provided in Chapter 4 of [8]. Cauchy's projection formula (see, for example, (A.43), p. 361 of [8]) says that

$$
b_{K}(u)=\frac{1}{2} \int_{S^{n-1}}|u \cdot v| d S(K, v),
$$

for all $u \in S^{n-1}$. Here $S(K, \cdot)$ is the surface area measure of $K$, defined for Borel subsets $E$ of $S^{n-1}$ by

$$
S(K, E)=V_{n-1}\left(g^{-1}(K, E)\right),
$$

where $g^{-1}(K, E)$ is the set of points in $\partial K$ at which there is an outer unit normal vector in $E$. Another useful formula is

$$
b_{K}(u)=n V(K, n-1 ;[o, u])
$$

for all $u \in S^{n-1}$, where the right-hand side is the mixed volume of $n-1$ copies of $K$ and one copy of the line segment $[o, u]$. See p. 360 and also Appendix A of [8] for an introduction to mixed volumes and the surface area measure.

A fundamental result for the present paper is Minkowski's existence theorem (see, for example, Theorem A.3.2 of [8]), which says that a Borel measure $\mu$ in $S^{n-1}$ is the surface area measure of some convex body $K$ in $\mathbb{R}^{n}$, unique up to translation, if and only if $\mu$ is not concentrated on any great sphere and

$$
\int_{S^{n-1}} u d \mu(u)=o .
$$

If $K$ is a convex body in $\mathbb{R}^{n}$, we denote the surface area of $K$ by $S(K)$. Cauchy's surface area formula (see (A.47), p. 361 of [8]) states that

$$
S(K)=\frac{1}{\kappa_{n-1}} \int_{S^{n-1}} b_{K}(u) d u .
$$

The isoperimetric inequality (see (B.12), p. 370 of [8]) can be written

$$
\left(\frac{S(K)}{n \kappa_{n}}\right)^{n} \geq\left(\frac{V(K)}{\kappa_{n}}\right)^{n-1} .
$$


The Hausdorffdistance $\delta(K, L)$ between two convex bodies in $\mathbb{R}^{n}$ can be conveniently defined by

$$
\delta(K, L)=\left\|h_{K}-h_{L}\right\|_{\infty} .
$$

The treatise of Schneider [25] is an excellent general reference for all of these topics.

The following deep result was proved independently by Campi [4] (for $n=3$ ) and Bourgain and Lindenstrauss [2]. The latter authors state their theorem in terms of a metric other than the Hausdorff metric, and make an additional assumption on the distance between the projection bodies. Groemer [14, Theorem 5.5.7] presents the version below that is more suitable for our purposes, and his proof yields the estimate of the constant in (7). This involves some tedious calculations (see http://www. ac. wwu . edu/ gardner; no attempt was made to obtain the optimal expression). In (7) and throughout the paper, the "big O" notation is used in the sense of "less than a constant multiple depending only on $n . "$

Proposition 2.1. Let $K$ and $L$ be origin-symmetric convex bodies in $\mathbb{R}^{n}, n \geq 3$, such that

$$
r_{0} B \subset K, L \subset R_{0} B
$$

for some $0<r_{0}<R_{0}$. If $0<a<2 /(n(n+4))$, there is a constant $c=c\left(a, n, r_{0}, R_{0}\right)$ such that

$$
\delta(K, L) \leq c \delta(\Pi K, \Pi L)^{a} .
$$

Moreover, if $0<a<2 /(n(n+4))$ is fixed, $r_{0}<1$, and $R_{0}>1$, then

$$
c=O\left(r_{0}^{-2 n-1} R_{0}^{5}\right) .
$$

Let $U=\left\{u_{i} \in S^{n-1}: i=1, \ldots, k\right\}$. The nodes corresponding to $U$ are defined as follows. The hyperplanes $u_{i}^{\perp}, i=1, \ldots, k$, partition $\mathbb{R}^{n}$ into a finite set of polyhedral cones, which intersect $S^{n-1}$ in a finite set of regions. The nodes $\pm v_{j} \in S^{n-1}, j=1, \ldots, l$, are the vertices of these regions. Thus, when $n=2$, the nodes are simply the $2 k$ unit vectors, each of which is orthogonal to some $u_{i}, i=1, \ldots, k$. When $n=3$, each $v_{j}$ is of the form $\left(u_{i} \times u_{i^{\prime}}\right) /\left\|u_{i} \times u_{i^{\prime}}\right\|$, where $1 \leq i<i^{\prime} \leq k$, and $l \leq k(k-1) / 2$. In general, $l=O\left(k^{n-1}\right)$.

Campi et al. [5] proved the following result.

Proposition 2.2. Let $K$ be a convex body in $\mathbb{R}^{n}$ and let $U=\left\{u_{i} \in S^{n-1}: i=1, \ldots, k\right\}$ span $\mathbb{R}^{n}$. Among all convex bodies with the same brightness function values as $K$ in the directions in $U$, there is a unique origin-symmetric convex polytope $P$, of maximal volume and with each of its facets orthogonal to one of the nodes corresponding to $U$.

\section{Reconstruction from Surface Area Measures}

By its definition (2), the surface area measure of a convex polytope in $\mathbb{R}^{n}$ whose facets have volumes $a_{j}$ and outer unit normals $v_{j}, j=1, \ldots, m$, is simply the sum of point 
masses of weight $a_{j}$ at $v_{j}$. Therefore Minkowski's existence theorem implies that given reals $a_{j}>0$ and unit vectors $v_{j}, j=1, \ldots, m$, that span $\mathbb{R}^{n}$ and satisfy

$$
\sum_{j=1}^{m} a_{j} v_{j}=o
$$

there is a convex polytope $P$ in $\mathbb{R}^{n}$ with $\operatorname{dim} P=n$ whose facets have volumes $a_{j}$ and outer normals $v_{j}, j=1, \ldots, m$. The following algorithm for reconstructing such a $P$ was proposed and implemented (at least for $n=3$ ) by Lemordant et al. [22].

\section{Algorithm MinkData}

Input: Natural numbers $n \geq 2$ and $m \geq n$; real numbers $a_{j}>0$ and directions $v_{j} \in S^{n-1}, j=1, \ldots, m$, that span $\mathbb{R}^{n}$ and satisfy (8).

Task: Compute the $k$-dimensional faces, $k=0, \ldots, n-1$, of a convex polytope $P$ in $\mathbb{R}^{n}$ whose facets have volumes $a_{j}$ and outer normals $v_{j}$, $j=1, \ldots, m$.

Action: If $n=2$ :

Order the directions $v_{j}$ so that the corresponding polar angles are increasing with $j$. Let $x_{0}=o$ and for $j=1, \ldots, m$ let $x_{j}=x_{j-1}+a_{j} v_{j}$. In view of (8), $x_{n}=o$ and so $\left\{x_{j}: j=1, \ldots, m\right\}$ form the vertices of a convex polygon $P^{\prime}$ with edges of length $a_{j}$ parallel to the directions $v_{j}, j=1, \ldots, m$. The output polygon $P$ is $P^{\prime}$ rotated by $\pi / 2$ about $o$.

Otherwise, if $n \geq 3$ :

1. For real numbers $h_{j}>0, j=1, \ldots, m$, let $h=\left(h_{1}, \ldots, h_{m}\right)$ and let $P(h)$ be the convex hull of the half-spaces with outer normals $v_{j}$ and bounding hyperplanes at distances $h_{j}$ from the origin, $j=1, \ldots, m$. Let

$$
\bar{a}=\sum_{j=1}^{m} a_{j}
$$

With initial guess $h^{(0)}=(1 / \bar{a}, 1 / \bar{a}, \ldots, 1 / \bar{a})$, if needed, solve the nonlinear optimization problem (NL):

$$
\begin{gathered}
\max _{h} V(P(h))^{1 / n}, \\
\text { subject to } \quad \sum_{j=1}^{m} a_{j} h_{j}=1 \\
\text { and } \quad h_{j} \geq 0, \quad j=1, \ldots, m .
\end{gathered}
$$

Let $\hat{h}=\left(\hat{h}_{1}, \ldots, \hat{h}_{m}\right)$ be a solution to (NL), and let

$$
\tilde{h}=(n V(P(\hat{h})))^{-1 /(n-1)} \hat{h}
$$

and $P=P(\tilde{h})$.

2. Compute the $k$-dimensional faces of $P$ for $k=0, \ldots, n-1$. 
A few remarks concerning Algorithm MinkData are appropriate. Firstly, it is an adaptation of the earlier algorithm of Little [23], which was based on Minkowski's original proof of his existence theorem (see Theorem 7.1.1 of [25]). This proof shows that the vector of distances from the origin to the hyperplanes containing the facets of the convex polytope with facets of volumes $a_{j}$ and outer normals $v_{j}, j=1, \ldots, m$, is, up to a scaling constant, the solution of the optimization problem $\left(\mathrm{NL}^{\prime}\right)$ :

$$
\begin{gathered}
\min _{h} \sum_{j=1}^{m} a_{j} h_{j}, \\
\text { subject to } \quad V(P(h))=1 \\
\text { and } \quad h_{j} \geq 0, \quad j=1, \ldots, m .
\end{gathered}
$$

The transition from $\left(\mathrm{NL}^{\prime}\right)$ to the equivalent problem $(\mathrm{NL})$ is achieved by programming duality; see Section 2.4 of [22]. In problem (NL), the objective function in (9) is concave by the Brunn-Minkowski inequality (see, for example, Section B.2 of [8] or Section 6.1 of [25]). Therefore (NL) involves a concave objective function and linear constraints. As is the case for $\left(\mathrm{NL}^{\prime}\right)$, the solution only gives the vector of distances from the origin to the hyperplanes of the desired polytope up to a scaling factor, which however is easy to calculate and is given above in Step 1.

The computation of the volume $V(P(h))$ for the objective function in (9) must be carried out at each step of the optimization problem (NL). The polytope $P(h)$ is known in its $\mathcal{H}$-representation, that is, by the equations of the hyperplanes containing its facets. Therefore $V(P(h))$ can be calculated by Lasserre's algorithm (see [19]).

The output polytope $P$ is also known in its $\mathcal{H}$-representation. Step 2 can be implemented by first computing the vertices of $P$, that is, its $\mathcal{V}$-representation, from its $\mathcal{H}$-representation, and then computing the convex hull of $P$ from its $\mathcal{V}$-representation. An algorithm for this purpose is described by Barber et al. [1].

Büeler et al. [3] provide an in-depth survey of techniques and extensive remarks concerning polytope volume computation and related matters concerning their $\mathcal{H}$ - and $\mathcal{V}$-representations.

As was mentioned in the Introduction, other algorithms for reconstruction from surface area measures can be found in [18] and [26]. For more details concerning the polynomial-time algorithm in [11], see Section 7.

\section{Some Lemmas}

This section contains some technical lemmas that we need for the algorithms in the next section.

Lemma 4.1. Let $R>0$, let $0<\varepsilon<1$, and let $U$ be an $\varepsilon$-net in $S^{n-1}$. Suppose that $K$ and $L$ are compact convex sets in $\mathbb{R}^{n}$ such that $h_{K}(u)=h_{L}(u) \leq R$ for each $u \in U$. Then

$$
\delta(K, L) \leq \frac{2 \varepsilon R}{1-\varepsilon}
$$


Proof. Let $u^{*}$ be the point in $S^{n-1}$ where $h_{K}$ attains its maximum. Let $u_{0}$ be the point in $U$ nearest to $u^{*}$ and let $w_{0}=\left(u^{*}-u_{0}\right) /\left\|u^{*}-u_{0}\right\|$. Then the subadditivity and positive homogeneity of $h_{K}$ (see p. 16 of [8]) gives

$$
h_{K}\left(u^{*}\right) \leq h_{K}\left(u_{0}\right)+h_{K}\left(u^{*}-u_{0}\right)=h_{K}\left(u_{0}\right)+\left\|u^{*}-u_{0}\right\| h_{K}\left(w_{0}\right) \leq R+\varepsilon h_{K}\left(u^{*}\right) .
$$

Therefore, for all $u \in S^{n-1}$,

$$
h_{K}(u) \leq h_{K}\left(u^{*}\right) \leq \frac{R}{1-\varepsilon} .
$$

The same argument shows that $h_{L}(u) \leq R /(1-\varepsilon)$ for each $u \in U$.

Let $u \in S^{n-1}$, let $u_{1}$ be the point in $U$ nearest to $u$, and let $w_{1}=\left(u-u_{1}\right) /\left\|u-u_{1}\right\|$. Using the subadditivity and positive homogeneity of $h_{K}$ and $h_{L}$, we obtain

$$
\begin{aligned}
h_{K}(u)-h_{L}(u) & \leq h_{K}\left(u_{1}\right)+h_{K}\left(u-u_{1}\right)-\left(h_{L}\left(u_{1}\right)-h_{L}\left(u_{1}-u\right)\right) \\
& =h_{K}\left(u-u_{1}\right)+h_{L}\left(u_{1}-u\right) \\
& =\left\|u-u_{1}\right\|\left(h_{K}\left(w_{1}\right)+h_{L}\left(-w_{1}\right)\right) \leq \frac{2 \varepsilon R}{1-\varepsilon} .
\end{aligned}
$$

The same bound applies to $h_{L}(u)-h_{K}(u)$, and this proves the lemma.

Lemma 4.2. Let $0<r<R$, let $0<\varepsilon<r^{n-1} /\left(5 R^{n-1}\right)$, and let $U$ be an $\varepsilon$-net in $S^{n-1}$. Let $K$ and $L$ be origin-symmetric convex bodies such that $r B \subset K \subset R B$ and $b_{K}(u)=b_{L}(u)$ for each $u \in U$. Then $r_{0} B \subset L \subset R_{0} B$, where

$$
R_{0}=\frac{3 n \kappa_{n}}{\kappa_{n-1}}\left(\frac{3}{2}\right)^{1 /(n-1)} \frac{R^{n}}{r^{n-1}} \quad \text { and } \quad r_{0}=\frac{\kappa_{n-1} r^{n-1}}{2^{n} R_{0}^{n-2}}
$$

Proof. The assumptions $r B \subset K \subset R B$ and $b_{K}(u)=b_{L}(u)$ for each $u \in U$ imply that

$$
s=\kappa_{n-1} r^{n-1} \leq h_{\Pi K}(u)=h_{\Pi L}(u) \leq \kappa_{n-1} R^{n-1}=t,
$$

for all $u \in U$. Since $\varepsilon<s /(5 t)$, Lemma 4.1 applied with $K, L$, and $R$ replaced by $\Pi K$, $\Pi L$, and $t$, respectively, gives

$$
\delta(\Pi K, \Pi L) \leq \frac{2 \varepsilon t}{1-\varepsilon}<\frac{s}{2} .
$$

From this and $s B \subset \Pi K \subset t B$ we obtain

$$
\frac{s}{2} B \subset \Pi L \subset \frac{3 t}{2} B
$$

Cauchy's surface area formula (4) now yields

$$
S(L)=\frac{1}{\kappa_{n-1}} \int_{S^{n-1}} b_{L}(u) d u \leq \frac{3 n \kappa_{n} t}{2 \kappa_{n-1}} .
$$


From the isoperimetric inequality (5), it follows that

$$
V(L) \leq \kappa_{n}\left(\frac{3 t}{2 \kappa_{n-1}}\right)^{n /(n-1)} .
$$

Let $x \in \partial L$, and let $u=x /\|x\|$. Then basic properties of mixed volumes (see (A.18), p. 355, and (A.26), p. 357 of [8]) yield

$$
V(L, n-1 ;[o, x]) \leq V(L, n-1 ; L)=V(L) .
$$

Using this, (3), (16), and the linearity property [8, (A.16), p. 355] of mixed volumes, we obtain

$$
\frac{s}{2}\|x\| \leq\|x\| b_{L}(u)=n V(L, n-1 ;[o, x]) \leq n V(L) .
$$

Consequently,

$$
\|x\| \leq \frac{2 n}{s} V(L)=R_{0},
$$

where $R_{0}$ is given by (15), and hence $L \subset R_{0} B$.

Let $c$ be the largest number such that $c B \subset L$. There are common parallel supporting hyperplanes to $c B$ and $L$ at contact points $z,-z \in \partial(c B) \cap \partial L$. Let $S$ be the closed slab bounded by these hyperplanes, and note that $L \subset S \cap R_{0} B$. If $u$ is any direction orthogonal to $z$, then $\left(S \cap R_{0} B\right) \mid u^{\perp} \subset E$, where $E$ is an $(n-1)$-dimensional box, the product of $n-1$ mutually orthogonal edges, with one edge parallel to $z$ of length $2 c$ and $n-2$ edges orthogonal to $z$, each of length $2 R_{0}$. Therefore

$$
\frac{s}{2} \leq b_{L}(u) \leq 2^{n-1} c R_{0}^{n-2},
$$

so $r_{0} B \subset L$, where $r_{0}$ is as in (15).

Lemma 4.3. Let $0<r<R$, let $0<\varepsilon<r^{n-1} /\left(5 R^{n-1}\right)$, and let $U$ be an $\varepsilon$-net in $S^{n-1}$. Let $K$ be an origin-symmetric convex body in $\mathbb{R}^{n}$ such that $r B \subset K \subset R B$, let $m \in \mathbb{N}$ be even, and let $a_{j}>0$ and $v_{j} \in S^{n-1}, j=1, \ldots, m$, be such that $a_{j}=a_{j+m / 2}$ and $v_{j+m / 2}=-v_{j}$ for $j=1, \ldots, m / 2$, and

$$
b_{K}(u)=\sum_{j=1}^{m} a_{j}\left|u \cdot v_{j}\right|
$$

for each $u \in U$. Then the vectors $v_{j}, j=1, \ldots, m$, span $\mathbb{R}^{n}$.

Proof. Suppose that the assumptions of the lemma hold but that the span of the vectors $v_{j}, j=1, \ldots, m$, is a $q$-dimensional subspace $S$ in $\mathbb{R}^{n}$ for some $q=1, \ldots, n-1$. Since $r B \subset K \subset R B$, we have

$$
s=\kappa_{n-1} r^{n-1} \leq h_{\Pi K}(u)=h_{Z}(u) \leq \kappa_{n-1} R^{n-1}=t,
$$


for $u \in U$, where

$$
h_{Z}(u)=\sum_{j=1}^{m} a_{j}\left|u \cdot v_{j}\right|,
$$

for $u \in S^{n-1}$, is the support function of a $q$-dimensional origin-symmetric zonotope (i.e., a finite vector sum of line segments; see, for example, p. 133 of [8]) in $S$. Lemma 4.1 applied with $K, L$, and $R$ replaced by $\Pi K, Z$, and $t$, respectively, gives

$$
\delta(\Pi K, Z) \leq \frac{2 \varepsilon t}{1-\varepsilon}<\frac{s}{2} .
$$

Since $s B \subset \Pi K$, we have $(s / 2) B \subset Z$, which is impossible.

\section{Reconstruction from Brightness Functions}

Our first two algorithms attempt to construct an origin-symmetric convex polytope whose brightness function values are equal to those of a given origin-symmetric convex body in a prescribed finite set of directions.

\section{Algorithm BrightLSQ}

Input: Natural numbers $n \geq 2$ and $k \geq n$; mutually nonparallel directions $u_{i} \in S^{n-1}, i=1, \ldots, k$, that span $\mathbb{R}^{n}$; brightness function values $b_{K}\left(u_{1}\right), \ldots, b_{K}\left(u_{k}\right)$ of an unknown origin-symmetric convex body $K$ in $\mathbb{R}^{n}$.

Task: Construct an origin-symmetric convex polytope $P$ in $\mathbb{R}^{n}$ such that $b_{P}\left(u_{i}\right)=b_{K}\left(u_{i}\right), i=1, \ldots, k$.

Action:

Phase I:

1. Calculate the nodes (see Section 2) $\pm v_{j} \in S^{n-1}, j=1, \ldots, l$, corresponding to $u_{i} \in S^{n-1}, i=1, \ldots, k$.

2. Write $\alpha=\left(\alpha_{1}, \ldots, \alpha_{l}\right)$ when $\alpha_{j} \geq 0, j=1, \ldots, l$. Solve the following constrained linear least squares problem (LLS):

$$
\begin{gathered}
\min _{\alpha} \sum_{i=1}^{k}\left(b_{K}\left(u_{i}\right)-\sum_{j=1}^{l} \alpha_{j}\left|u_{i} \cdot v_{j}\right|\right)^{2}, \\
\text { subject to } \quad \alpha_{j} \geq 0, \quad j=1, \ldots, l .
\end{gathered}
$$

Let $\hat{\alpha}_{j}, j=1, \ldots, l^{\prime}, l^{\prime} \leq l$, be a suitably relabeled solution to (LLS) in which any $\hat{\alpha}_{j}$ with $\hat{\alpha}_{j}=0$ has been discarded. Let $m=2 l^{\prime}$, and let $a_{j}=a_{j+m / 2}=\hat{\alpha}_{j}$ and $v_{j+m / 2}=-v_{j}$ for $j=1, \ldots, m / 2$. Then (8) is satisfied. If the vectors $v_{j}, j=1, \ldots, m$, span $\mathbb{R}^{n}$, Minkowski's existence theorem implies that there is a unique origin-symmetric convex polytope $P$ with facets of volumes $a_{j}$ and outer normals $v_{j}, j=1, \ldots, m$.

Phase II:

3. Use Algorithm MinkData to reconstruct $P$ from $a_{j}$ and $v_{j}, j=$ $1, \ldots, m$. 
The following algorithm, due to Markus Kiderlen, achieves the same purpose as Algorithm BrightLSQ by a linear programming approach.

\section{Algorithm BrightLP}

The input and task for this algorithm are as in Algorithm BrightLSQ. It proceeds in the same way except that in Step 2, (LLS) is replaced by the following linear program (LP):

$$
\begin{gathered}
\min _{\alpha} \sum_{i=1}^{k}\left(b_{K}\left(u_{i}\right)-\sum_{j=1}^{l} \alpha_{j}\left|u_{i} \cdot v_{j}\right|\right), \\
\text { subject to } \quad \sum_{j=1}^{l} \alpha_{j}\left|u_{i} \cdot v_{j}\right| \leq b_{K}\left(u_{i}\right), \quad i=1, \ldots, k, \\
\text { and } \quad \alpha_{j} \geq 0, \quad j=1, \ldots, l .
\end{gathered}
$$

The next algorithm attempts to construct from the data an origin-symmetric convex polytope with less than or equal to a prescribed even number $N$ of facets.

\section{Algorithm BrightNFacets}

Input: Natural numbers $n \geq 2, N$ even with $N \geq 2 n$, and $k \geq N / 2$; mutually nonparallel directions $u_{i} \in S^{n-1}, i=1, \ldots, k$, that span $\mathbb{R}^{n}$; brightness function values $b_{K}\left(u_{1}\right), \ldots, b_{K}\left(u_{k}\right)$ of an unknown origin-symmetric convex body $K$ in $\mathbb{R}^{n}$.

Task: Construct an origin-symmetric convex polytope $P$ in $\mathbb{R}^{n}$ with less than or equal to $N$ facets and such that $b_{P}\left(u_{i}\right)=b_{K}\left(u_{i}\right), i=1, \ldots, k$.

\section{Action:}

Phase I:

1. If $\alpha_{j} \geq 0$ and $\omega_{j} \in S^{n-1}, j=1, \ldots, N / 2$, let $\alpha=\left(\alpha_{1}, \ldots, \alpha_{N / 2}\right)$ and $\omega=\left(\omega_{1}, \ldots, \omega_{N / 2}\right)$. Solve the following constrained nonlinear least squares problem (NLS):

$$
\begin{gathered}
\min _{(\alpha, \omega)} \sum_{i=1}^{k}\left(b_{K}\left(u_{i}\right)-\sum_{j=1}^{N / 2} \alpha_{j}\left|u_{i} \cdot \omega_{j}\right|\right)^{2}, \\
\text { subject to } \quad \alpha_{j} \geq 0, \quad j=1, \ldots, N / 2 \\
\text { and } \omega_{j} \in S^{n-1}, \quad j=1, \ldots, N / 2 .
\end{gathered}
$$

Let $(\hat{\alpha}, \hat{\omega})$, where $\hat{\alpha}=\left(\hat{\alpha}_{1}, \ldots, \hat{\alpha}_{m / 2}\right)$ and $\hat{\omega}=\left(\hat{\omega}_{1}, \ldots, \hat{\omega}_{m / 2}\right), m \leq N$, be a suitably relabeled solution to (NLS) in which each $\hat{\alpha}_{j}$ and $\hat{\omega}_{j}$ with $\hat{\alpha}_{j}=0$ has been discarded. Let $a_{j}=a_{j+m / 2}=\hat{\alpha}_{j}, v_{j}=\hat{\omega}_{j}$, and $v_{j+m / 2}=-\hat{\omega}_{j}$, $j=1, \ldots, m / 2$. These definitions and the constraints (24) and (25) ensure that $a_{j}>0$ and $v_{j} \in S^{n-1}, j=1, \ldots, m$, and that (8) holds. If the vectors $v_{j}, j=1, \ldots, m$, span $\mathbb{R}^{n}$, Minkowski's existence theorem implies that 
there is a unique origin-symmetric convex polytope $P$ with facets of volumes $a_{j}$ and outer normals $v_{j}, j=1, \ldots, m$.

Phase II:

2. Use Algorithm MinkData to reconstruct $P$ from $a_{j}$ and $v_{j}, j=$ $1, \ldots, m$.

Remarks concerning the assumption that the unknown convex body $K$ is origin symmetric can be found in Sections 6 and 10. As stated, the algorithms in this section may not succeed in their tasks, since Phase II cannot be performed if the output vectors $v_{j}$ from Phase I lie in a proper subspace of $\mathbb{R}^{n}$. In practice, of course, one can run the algorithms and simply discard the output of Phase I in the case of such a degeneracy. However, the following theorem provides some reasonable extra conditions on the input which guarantees that the algorithms will succeed.

Theorem 5.1. Suppose that $0<r<R, U=\left\{u_{i} \in S^{n-1}: i=1, \ldots, k\right\}$ is a set of mutually nonparallel directions such that $U \cup-U$ is an $\varepsilon$-net with $0<\varepsilon<$ $r^{n-1} /\left(5 R^{n-1}\right)$, and $K$ is an origin-symmetric convex body in $\mathbb{R}^{n}$ with $r B \subset K \subset R B$. Then Algorithms BrightLSQ and BrightLP succeed in their tasks, and if in addition $K$ is a convex polytope with $N$ facets, Algorithm BrightNFacets succeeds in its task.

Proof. By Proposition 2.2, there is an origin-symmetric convex polytope with the same brightness function measurements as $K$ in the input directions, each of whose facets has outer unit normal $v_{j}$ for some $j=1, \ldots, m$. Since the output $a_{j}$ and $v_{j}, j=1, \ldots, m$, of Phase I of Algorithm BrightLSQ could be the volumes and outer unit normals of the facets of this polytope, the optimal value of the objective function in (18) is zero.

The output $a_{j}$ and $v_{j}, j=1, \ldots, m$, from Phase I of Algorithm BrightLSQ also constitute a possible output of Phase I of Algorithm BrightLP, because these values satisfy the constraint (21) in the program (LP) with equality; they also give a zero optimal objective function value in (20).

If the unknown convex body $K$ in Algorithm BrightNFacets is a convex polytope with $N$ facets, then the output $a_{j}$ and $v_{j}, j=1, \ldots, m$, of Phase I could be the volumes and outer unit normals of the facets of this polytope. Therefore the optimal value of the objective function in (23) is zero.

For each algorithm, therefore, the optimal objective function value in the corresponding optimization program is zero. Therefore (17) holds in each case. Lemma 4.3 implies that the vectors $v_{j}, j=1, \ldots, m$, span $\mathbb{R}^{n}$, and Phase II can be performed to construct the output polytope $P$. By (1) and (17), $b_{P}\left(u_{i}\right)=b_{K}\left(u_{i}\right)$ for $i=1, \ldots, k$.

\section{Proof of Convergence}

Theorem 6.1. Let $n \geq 2$, let $0<r<R$, and let $K$ be an origin-symmetric convex body in $\mathbb{R}^{n}$ such that $r B \subset K \subset R B$. Let $u_{i}, i \in \mathbb{N}$, be a sequence of mutually nonparallel directions such that $\left\{ \pm u_{i}\right\}$ is dense in $S^{n-1}$. For either Algorithm BrightLSQ or Algorithm BrightLP, let $P_{k}, k \geq n$, be an output, if any, to the algorithm corresponding to input directions $u_{i}, i=1, \ldots, k$. Then $\delta\left(K, P_{k}\right) \rightarrow 0$ as $k \rightarrow \infty$. 
Proof. For each $k$, let

$$
\Delta_{k}=\max _{u \in S^{n-1}} \min _{1 \leq i \leq k}\left\{\left\|u-u_{i}\right\|,\left\|u-\left(-u_{i}\right)\right\|\right\} .
$$

Then the set $U_{k}=\left\{u_{i}: i=1, \ldots, k\right\}$ is such that $U_{k} \cup-U_{k}$ is an $\varepsilon$-net in $S^{n-1}$, where $\varepsilon=\Delta_{k}$. Since $\left\{ \pm u_{i}\right\}$ is dense in $S^{n-1}, \Delta_{k} \rightarrow 0$ as $k \rightarrow \infty$, so we can choose an $N_{0}$ so that $\Delta_{k}<r^{n-1} /\left(5 R^{n-1}\right)$ for $k \geq N_{0}$. By Theorem 5.1, for $k \geq N_{0}$, Algorithm BrightLSQ or Algorithm BrightLP produce a convex polytope $P_{k}$ such that

$$
h_{\Pi K}( \pm u)=b_{K}( \pm u)=b_{P_{k}}( \pm u)=h_{\Pi P_{k}}( \pm u) \text {, }
$$

for $u \in U_{k}$.

By assumption $K \subset R B$, so $\Pi K \subset \kappa_{n-1} R^{n-1} B$. Using (27), we apply Lemma 4.1 with $U=U_{k}$ and $K, L$, and $R$ replaced by $\Pi K, \Pi P_{k}$, and $\kappa_{n-1} R^{n-1}$, respectively, to obtain

$$
\delta\left(\Pi K, \Pi P_{k}\right) \leq \frac{2 \Delta_{k} \kappa_{n-1} R^{n-1}}{1-\Delta_{k}}<\frac{5}{2} \Delta_{k} \kappa_{n-1} R^{n-1},
$$

for $k \geq N_{0}$.

Suppose that $n=2$. It is easy to see (see p. 130 of [8]) that the projection body $\Pi L$ of any origin-symmetric convex body $L$ is just $2 L$ rotated by $\pi / 2$ about $o$. From (28) it then follows directly that

$$
\delta\left(K, P_{k}\right)=\frac{1}{2} \delta\left(\Pi K, \Pi P_{k}\right) \leq \frac{5}{2} \Delta_{k} R,
$$

for $k \geq N_{0}$.

Now suppose that $n \geq 3$. From Lemma 4.2 with $U=U_{k}$ and $L=P_{k}$ we conclude that

$$
r_{0} B \subset P_{k} \subset R_{0} B,
$$

where $R_{0}$ and $r_{0}$ are given by (15), and these containments also hold for $K$ since $r_{0} \leq r$ and $R \leq R_{0}$. By (6), (15), (28), and (30), for each $0<a<2 /(n(n+4))$ there is a $c^{\prime}=c^{\prime}(a, n, r, R)>0$ such that

$$
\delta\left(K, P_{k}\right) \leq c^{\prime} \Delta_{k}^{a},
$$

for $k \geq N_{0}$.

The estimates (29) and (31) imply that for $n \geq 2, \delta\left(K, P_{k}\right) \rightarrow 0$ as $k \rightarrow \infty$.

Exactly the same proof yields the following theorem, with the same estimates for convergence.

Theorem 6.2. Let $n \geq 2$ and $N$ be natural numbers with $N$ even and $N \geq 2 n$. Let $0<r<R$, and let $K$ be an origin-symmetric convex polytope in $\mathbb{R}^{n}$ with $N$ facets satisfying $r B \subset K \subset R B$. Suppose that $u_{i}, i \in \mathbb{N}$, is a sequence of mutually nonparallel directions such that $\left\{ \pm u_{i}\right\}$ is dense in $S^{n-1}$. Let $P_{k}, k \geq n$, be an output, if any, to Algorithm BrightNFacets corresponding to input directions $u_{i}, i=1, \ldots, k$. Then $\delta\left(K, P_{k}\right) \rightarrow 0$ as $k \rightarrow \infty$. 
The proof above of Theorem 6.1 can be shortened somewhat, but we have included bounds that are useful in estimating the speed of convergence; indeed, they will be needed in the proof of Theorem 7.2 below. These estimates show that for a fixed $k$ it is desirable to choose the input directions $u_{i}, i=1, \ldots, k$, in order to minimize $\Delta_{k}$. When $n=2$, one can take these directions with equally spaced polar angles in the interval $[0, \pi)$, so that $\Delta_{k}=\pi / k$ and

$$
\delta\left(K, P_{k}\right) \leq \frac{5 \pi R}{2 k}
$$

when $k>5 \pi R / r$. As Kiderlen [17] also notes, for $n \geq 3$ and general $k$, it is not known how to minimize $\Delta_{k}$, but special cases of this problem are solved; see [7].

A separate proof of convergence for Algorithm BrightLP can be based on a result of Campi et al. [6]. This says that for any zonoid and finite set of directions, there is a zonotope that has the same support function values as the zonoid in those directions. Since every projection body is a zonoid (see Theorem 4.1.11 of [8]), this result can be applied to $\Pi K$ to obtain (27). Kiderlen [17] also utilizes the result of Campi et al. and we thank him for bringing to our attention its applicability here.

The assumption of origin symmetry in our convergence theorems is necessary, since $-K$ has the same brightness function as $K$. The lack of uniqueness is actually more serious. To see this, let $K$ be a convex body such that $-K \neq K$. Let $K_{t}$ be a convex body which, by Minkowski's existence theorem, can be defined by

$$
S\left(K_{t}, \cdot\right)=(1-t) S(K, \cdot)+t S(-K, \cdot)
$$

for $0 \leq t \leq 1$. Then $b_{K_{t}}=b_{K}$ for $0 \leq t \leq 1$, by (1). By the Kneser-Süss inequality (see Theorem 7.1.3 of [25]), $V\left(K_{t}\right)$ is strictly increasing for $0 \leq t \leq \frac{1}{2}$ (and strictly decreasing for $\frac{1}{2} \leq t \leq 1$ ), so there are infinitely many mutually noncongruent convex bodies whose brightness function equals that of $K$. Also, the existence of nonspherical convex bodies of constant brightness (see, for example, Section 3.3 of [8]) shows that even the unit ball is not determined among all convex bodies, up to translation, by its brightness function. In fact, only origin-symmetric parallelotopes are determined in this sense; see [9].

Let $U$ be any finite set of directions in $S^{n-1}$, and let $K$ be an origin-symmetric convex polytope in $\mathbb{R}^{n}$ with at least one facet not orthogonal to any of the nodes corresponding to $U$. Proposition 2.2 implies that there is an origin-symmetric convex polytope $P$ with the same brightness function as $K$ in the directions in $U$ and facets orthogonal to these nodes. Therefore $P$ is not a translate of $K$. Furthermore, let $U$ be an arbitrary, possibly infinite, set of directions such that $U \cup-U$ is not dense in $S^{1}$. Then there exists an open arc $W$ in $S^{1}$ such that $(U \cup-U) \cap W=\emptyset$. Let $w_{1}, w_{2}$ be the endpoints of $W$, let $w \in W$, and let $Q$ be the parallelogram with diagonal $[-w, w]$ and edges parallel to $w_{1}$ and $w_{2}$. Any origin-symmetric convex $m$-gon contained in $Q$ and with vertices at $-w$ and $w$ has the same brightness function as the line segment $[-w, w]$ in all the directions in $U$. Therefore equality of brightness functions, even in a large set of directions, is not sufficient to guarantee equality of origin-symmetric convex bodies, even if these are polytopes with equal numbers of facets. It also follows that the hypothesis on the sequence $\left\{u_{i}\right\}$ of directions in Theorems 6.1 and 6.2 is in general necessary. 
Origin symmetry is not required in Algorithm MinkData. Despite the remarks above, there are also modified versions of Algorithms BrightLSQ, BrightLP, and BrightNFacets, and Theorems 6.1 and 6.2, for which the origin symmetry of $K$ is not required. Indeed, if $K$ is an arbitrary convex body, its Blaschke body $\nabla K$, defined by (32) with $t=\frac{1}{2}$, is the unique origin-symmetric convex body with the same brightness function. Since $b_{\nabla K}=b_{K}$, the proofs of Theorems 6.1 and 6.2 show that under the same assumptions, the outputs of the modified forms of Algorithms BrightLSQ, BrightLP, and BrightNFacets, that accept as input an arbitrary convex body $K$, converge to $\nabla K$ in the Hausdorff metric.

Further remarks about disposing with origin symmetry can be found in Section 10.

\section{An Oracle-Polynomial-Time Algorithm}

In this section we describe an oracle-polynomial-time algorithm for reconstructing an approximation to an origin-symmetric rational convex polytope of full and fixed dimension from its brightness function. Such complexity results are somewhat technical, but we attempt to keep the description brief by referring to other sources.

We begin with the problem $n$-MinkApp.

Instance: $m \in \mathbb{N}$; nonzero rational vectors $z_{j}, j=1, \ldots, m$, no two positively dependent, that span $\mathbb{R}^{n}$; positive rationals $c_{j}, j=1, \ldots, m$, such that

$$
\sum_{j=1}^{m} c_{j} z_{j}=o
$$

a rational error bound $\varepsilon_{0}>0$.

Task: Determine a rational vector $\bar{b}=\left(\bar{b}_{1}, \ldots, \bar{b}_{m}\right)$ such that the volume $\bar{a}_{j}$ of the facet $\left\{x \in \bar{P}: x \cdot z_{j}=\bar{b}_{j}\right\}$ of the polytope

$$
\bar{P}=\left\{x \in \mathbb{R}^{n}: A x \leq \bar{b}\right\},
$$

where $A$ is the matrix whose rows are $z_{j}, j=1, \ldots, m$, satisfies

$$
\left|c_{j}\left\|z_{j}\right\|-\bar{a}_{j}\right| \leq \varepsilon_{0} .
$$

Gritzmann and Hufnagel [11, Theorem 1] proved that this problem can be solved in polynomial time. They employ the standard binary Turing machine model in which the input data is encoded in binary form, and the performance of the algorithm is measured in terms of the number of operations of a Turing machine. In this model, all input and output must be rational. Neither unit normals nor facet volumes of a rational convex polytope (i.e., a polytope with rational vertices) need be rational. Therefore an instance of $n$-MinkApp takes rational vectors $z_{j}$ and rationals $c_{j}$ that correspond to unit normals $z_{j} /\left\|z_{j}\right\|$ and facet volumes $c_{j}\left\|z_{j}\right\|$ of a rational polytope

$$
P=\left\{x \in \mathbb{R}^{n}: A x \leq b\right\} .
$$

The output is the $\mathcal{H}$-representation of a polytope $\bar{P}$ that has the same outer unit normals as $P$ and whose facet volumes are within the prescribed error bound $\varepsilon_{0}$ of those of $P$. 
We wish to formulate a similar problem for which the output corresponds to an origin-symmetric rational convex polytope that approximates an unknown one $P$ that is only accessible via its brightness function. Note that even for rational polytopes $P$ and rational vectors $w, b_{P}(w /\|w\|)$ is not necessarily rational. However, by (3) and the linearity property $[8,($ A.16), p. 355] of mixed volumes,

$$
b_{P}\left(\frac{w}{\|w\|}\right)=n V\left(P, n-1 ;\left[o, \frac{w}{\|w\|}\right]\right)=\frac{n}{\|w\|} V(P, n-1 ;[o, w])
$$

so $\|w\| b_{P}(w /\|w\|)$ is rational if $V(P, n-1 ;[o, w])$ is rational. Now the volume of a rational convex polytope is rational by the determinant formula, so by the so-called polarization formula [8, (A.8), p. 353], any mixed volume of rational polytopes is rational. This shows that $\|w\| b_{P}(w /\|w\|)$ is rational.

Clearly any representation of $P$ itself cannot be part of the input. To handle such situations, the notion of an oracle has been developed; see, for example, [12] and p. 26 of [15]. We assume that the unknown rational convex polytope $P$ in $\mathbb{R}^{n}$ is well bounded, that is, $r B \subset P \subset R B$ for some rational numbers $0<r<R$. The oracle we require has the following input and output:

Oracle input: A rational vector $w$ in $\mathbb{R}^{n}$.

Oracle output: The rational $\|w\| b_{P}(w /\|w\|)$.

The size of an integer $p$ is $\langle p\rangle=1+\left\lceil\log _{2}(|p|+1)\right\rceil$. The size of a rational $p / q$, where $p$ and $q$ are relatively prime, is $\langle p\rangle+\langle q\rangle$, and the size of a rational vector is the sum of the sizes of its components. The size $\langle P\rangle$ of a well-bounded rational convex polytope $P$ is defined to be the sum of $\langle r\rangle,\langle R\rangle$, and the sizes of the vertices of $P$. (Here we are assuming the oracle has access to $P$ in its $\mathcal{V}$-representation. This is not important, however, since a $\mathcal{V}$-representation can be converted to an $\mathcal{H}$-representation, and vice versa, in polynomial time when the dimension is fixed; see [12].) It is a consequence of 4.2.1 of [13] that when $n$ is fixed, $V(P, n-1 ;[o, w])$ and hence $\|w\| b_{P}(w /\|w\|)$ can be computed in a time bounded by a polynomial in $\langle P\rangle$ and $\langle w\rangle$.

Note that with our formulation of the oracle, it does not conform to the general assumption about oracles on p. 26 of [15] that the size of the oracle output is bounded by a polynomial in the size of the input. However, the unknown polytope $P$ is fixed and must be accessible to the oracle in order for it to produce an output, so it is reasonable to allow the size of this output to depend on $\langle P\rangle$ as well as $\langle w\rangle$.

With this background, we can now describe the algorithmic problem $n$-BrightApp.

Instance: Rationals $0<r<R$ and a rational error bound $\varepsilon>0$.

Task: Find a rational matrix $A$ and rational vector $\bar{b}$ such that

$$
\bar{P}=\left\{x \in \mathbb{R}^{n}: A x \leq \bar{b}\right\}
$$

is an origin-symmetric convex polytope in $\mathbb{R}^{n}$ satisfying $\delta(P, \bar{P})<\varepsilon$, where $P$ is an origin-symmetric rational convex polytope in $\mathbb{R}^{n}$ such that $r B \subset P \subset R B$, that is accessible only via the above brightness function oracle. 
We remind the reader that the "big $O$ " notation denotes "less than a constant multiple depending only on $n$." For the main result in this section we need the following lemma.

Lemma 7.1. For each rational $\varepsilon>0$ and $n \geq 2$, there is a set $W$ of $O\left(\varepsilon^{1-n}\right)$ rational vectors in $\mathbb{R}^{n}$ such that $U=\{w /\|w\|: w \in W\}$ is an $\varepsilon$-net in $S^{n-1}$ and $\langle w\rangle=O(\langle\varepsilon\rangle)$, for each $w \in W$.

Proof. We first prove by induction on $n$ that there is an $\varepsilon$-net $U^{\prime}(n, \varepsilon)$ of $O\left(\varepsilon^{1-n}\right)$ points in $S^{n-1}$. Clearly there is a set of $O\left(\varepsilon^{-1}\right)$ points in $S^{1}$ that form an $\varepsilon$-net. Suppose that $U^{\prime}(n-1, \varepsilon / 2)$ is an $(\varepsilon / 2)$-net in $S^{n-2}$ containing $O\left(\varepsilon^{2-n}\right)$ points. Let

$$
X=\left\{-1+j \varepsilon: j=0, \ldots,\left[\frac{2}{\varepsilon}\right]\right\}
$$

and $Y=U^{\prime}(n-1, \varepsilon / 2) \times X$. Then $X$ is an $(\varepsilon / 2)$-net in $[-1,1]$, so $Y$ is an $\varepsilon$-net in the cylinder $S^{n-2} \times[-1,1]$ containing $O\left(\varepsilon^{1-n}\right)$ points. The map of this cylinder into $S^{n-1}$ that takes $(1, u, z)$ to $\left(\sqrt{1-z^{2}}, u, z\right)$, where we use cylindrical coordinates, does not increase distances between points. The image $U^{\prime}(n, \varepsilon)$ of $Y$ under this map is therefore an $\varepsilon$-net in $S^{n-1}$ containing $O\left(\varepsilon^{1-n}\right)$ points.

Let $\varepsilon=p / q$, where $p$ and $q$ are relatively prime. For each $u_{0} \in U^{\prime}(n, \varepsilon / 4)$, let $w=w\left(u_{0}\right)$ be the nearest rational vector to $u_{0}$ that is not contained in $B$ and whose components all have denominators that are integer multiples of $n q$. The vectors $u_{0}$ and $w$ are contained in a cube, and therefore a ball $D$, of diameter $\sqrt{n} /(n q)=1 /(\sqrt{n} q)$. If $C$ is the cap in $S^{n-1}$ formed by intersecting the convex hull of $o$ and $D$ with $S^{n-1}$, then the diameter of $C$ is no larger than that of $D$, and $u_{0}$ and $w /\|w\|$ belong to $C$. Therefore $\left\|u_{0}-w /\right\| w\|\| \leq 1 /(\sqrt{n} q)$. Let $u \in S^{n-1}$ be arbitrary, and suppose, without loss of generality, that $u_{0}$ is the point in $U^{\prime}(n, \varepsilon / 4)$ nearest to $u$. Then

$$
\left\|u-\frac{w}{\|w\|}\right\| \leq\left\|u-u_{0}\right\|+\left\|u_{0}-\frac{w}{\|w\|}\right\| \leq \frac{\varepsilon}{4}+\frac{1}{\sqrt{n} q} \leq\left(\frac{1}{4}+\frac{1}{\sqrt{n}}\right) \varepsilon<\varepsilon .
$$

Therefore $W=\left\{w\left(u_{0}\right): u_{0} \in U^{\prime}(n, \varepsilon / 4)\right\}$ is a set of $O\left(\varepsilon^{1-n}\right)$ rational vectors in $\mathbb{R}^{n}$ such that $U=\{w /\|w\|: w \in W\}$ is an $\varepsilon$-net in $S^{n-1}$. It is clear from the definition of $W$ that for each $w \in W$ we have $\langle w\rangle=O(\langle\varepsilon\rangle)$.

The order of $\varepsilon$ in the previous lemma is optimal, but the proof does not provide the best-known constant, which apparently can be deduced from a result of Rogers [24].

We can now state the main result. In an oracle-polynomial time algorithm (see p. 27 of [15]) each call of the oracle counts one step. Of course, since the output of the oracle is used in the algorithm, its time depends also on the size of the unknown polytope $P$.

Theorem 7.2. For each fixed $n \in \mathbb{N}, n$-BrightApp can be solved in oracle-polynomial time.

Proof. Fix $n \in \mathbb{N}$ and let $\varepsilon>0$ be rational. For a suitable $q$ to be computed later, Lemma 7.1 implies that there is an $M$ so that with $k=M q^{n-1}$, there is a $(1 / q)$-net 
$U=\left\{u_{1}, \ldots, u_{k}\right\}$ in $S^{n-1}$, where each $u \in U$ is of the form $u=w /\|w\|$ for some rational vector $w$ such that $\langle w\rangle=O(\langle q\rangle)$. We first use Algorithm BrightLP and the above brightness function oracle with input rationals $w_{i}$ such that $u_{i}=w_{i} /\left\|w_{i}\right\|, i=1, \ldots, k$, to find outer normals and facet volumes of an origin-symmetric convex polytope $P_{k}$ such that $\delta\left(P, P_{k}\right)<\varepsilon / 2$. Then we run the polynomial-time algorithm for $n$-MinkApp, with a rational error bound $\varepsilon_{0}$, also to be computed later, small enough to ensure that the resulting origin-symmetric convex polytope $\bar{P}$ satisfies $\delta\left(P_{k}, \bar{P}\right)<\varepsilon / 2$ and hence $\delta(P, \bar{P})<\varepsilon$. (We remark that the algorithm for $n$-MinkApp in [11] also provides a Hausdorff metric approximation from estimates of facet volumes as an intermediate step.)

The size of the input to Algorithm BrightLP (the same as that for Algorithm BrightLSQ above) is bounded by $O(\langle q\rangle)$ and a polynomial in $\langle P\rangle$. Step 1 of Algorithm BrightLP involves the computation of the nodes $\pm v_{j}, j=1, \ldots, l$, corresponding to $U$. As we noted in Section 2, $l=O\left(k^{n-1}\right)$. Each $u \in U$ is of the form $u=w /\|w\|$ for some rational vector $w$ and the nodes $\pm v_{j}$ in each antipodal pair are parallel to a line that is the intersection of $n-1$ hyperplanes, each orthogonal to one of these rational vectors. Thus each such line can be computed by solving $n-1$ equations with rational coefficients, each of size $O(\langle q\rangle)$. To each node $v_{j}$, therefore, there corresponds a rational vector $z_{j}$ for which $v_{j}=z_{j} /\left\|z_{j}\right\|$ with $\left\langle z_{j}\right\rangle=O(\langle q\rangle)$ and which can be computed in time $O(\langle q\rangle)$.

We need to work with the following equivalent modification $\left(\mathrm{LP}^{\prime}\right)$ of (LP), whose input consists of the rationals $\left\|w_{i}\right\| b_{P}\left(w_{i} /\left\|w_{i}\right\|\right), i=1, \ldots, k$, and rational vectors $z_{j}$, $j=1, \ldots, l$, and whose variables are $\beta=\left(\beta_{1}, \ldots, \beta_{l}\right)$ :

$$
\begin{gathered}
\min _{\beta} \sum_{i=1}^{k}\left(\left\|w_{i}\right\| b_{P}\left(\frac{w_{i}}{\left\|w_{i}\right\|}\right)-\sum_{j=1}^{l} \beta_{j}\left|w_{i} \cdot z_{j}\right|\right), \\
\text { subject to } \quad \sum_{j=1}^{l} \beta_{j}\left|w_{i} \cdot z_{j}\right| \leq\left\|w_{i}\right\| b_{P}\left(\frac{w_{i}}{\left\|w_{i}\right\|}\right), \quad i=1, \ldots, k, \\
\text { and } \quad \beta_{j} \geq 0, \quad j=1, \ldots, l .
\end{gathered}
$$

The input to $\left(\mathrm{LP}^{\prime}\right)$ is bounded by $O(\langle q\rangle)$ and a polynomial in $\langle P\rangle$. As is well known, the linear program $\left(\mathrm{LP}^{\prime}\right)$ can be solved in polynomial time. Let $\hat{\beta}_{j}, j=1, \ldots, l^{\prime}, l^{\prime} \leq l$, be a suitably relabeled solution to $\left(\mathrm{LP}^{\prime}\right)$ in which any $\hat{\beta}_{j}$ with $\hat{\beta}_{j}=0$ has been discarded. Let $m=2 l^{\prime}$, let $c_{j}=c_{j+m / 2}=\hat{\beta}_{j}$, and let $v_{j+m / 2}=-v_{j}$ and $z_{j+m / 2}=-z_{j}$ for $j=1, \ldots, m / 2$. Then the rational vectors $z_{j}$ and rationals $c_{j}$ correspond to unit normals $v_{j}=z_{j} /\left\|z_{j}\right\|$ and facet volumes $c_{j}\left\|z_{j}\right\|$ of an origin-symmetric polytope $P_{k}$ such that $b_{P_{k}}\left( \pm u_{i}\right)=b_{P}\left( \pm u_{i}\right), i=1, \ldots, k$. Observe also that (33) is satisfied and hence the rational quantities $z_{j}$ and $c_{j}, j=1, \ldots, m$, form a suitable input to the problem $n$ MinkApp.

It remains to compute a suitable $q$ and $\varepsilon_{0}$. Following exactly the proof of Theorem 6.1 and choosing $a=1 /(n(n+4))$, we conclude that if $1 / q<r^{n-1} /\left(5 R^{n-1}\right)$, then

$$
\delta\left(P, P_{k}\right) \leq c\left(n, r_{0}, R_{0}\right)\left(\frac{5}{2 q} \kappa_{n-1} R^{n-1}\right)^{1 /(n(n+4))},
$$

where $R_{0}$ and $r_{0}$ are given by (15) and $c\left(n, r_{0}, R_{0}\right)$ is the constant from Proposition 2.1 corresponding to $a=1 /(n(n+4))$. Therefore we can ensure that $\delta\left(P, P_{k}\right)<\varepsilon / 2$ by 
choosing

$$
q>\max \left\{5\left(\frac{R}{r}\right)^{n-1}, \frac{5}{2} \kappa_{n-1} R^{n-1}\left(2 \varepsilon^{-1} c\left(n, r_{0}, R_{0}\right)\right)^{n(n+4)}\right\} .
$$

By (7), we can choose $q$ so that it is bounded by a polynomial in $r^{-1}, R$, and $\varepsilon^{-1}$.

To find a suitable estimate for $\varepsilon_{0}$, let $\bar{a}_{j}$ denote the volume of the $j$ th facet of the polytope $\bar{P}$ corresponding to the output of $n$-MinkApp for which the input quantities are $z_{j}$ and $c_{j}, j=1, \ldots, m$. By (1), for each $u \in S^{n-1}$ we have

$$
h_{\Pi P_{k}}(u)=b_{P_{k}}(u)=\sum_{j=1}^{m / 2}\left(c_{j}\left\|z_{j}\right\|\right)\left|u \cdot v_{j}\right|
$$

and

$$
h_{\Pi \bar{P}}(u)=b_{\bar{P}}(u)=\sum_{j=1}^{m / 2} \bar{a}_{j}\left|u \cdot v_{j}\right| .
$$

Therefore

$$
\left|b_{P_{k}}(u)-b_{\bar{P}}(u)\right| \leq \delta\left(\Pi P_{k}, \Pi \bar{P}\right) \leq \sum_{j=1}^{m / 2}\left|c_{j}\left\|z_{j}\right\|-\bar{a}_{j}\right|<\frac{m \varepsilon_{0}}{2},
$$

for all $u \in S^{n-1}$. The containment (30) holds for $P_{k}$, so

$$
s_{0}=\kappa_{n-1} r_{0}^{n-1} \leq b_{P_{k}}(u) \leq \kappa_{n-1} R_{0}^{n-1}=t_{0},
$$

for all $u \in S^{n-1}$, where $R_{0}$ and $r_{0}$ are given by (15). It follows, using (37), that if $\varepsilon_{0}<\kappa_{n-1} r_{0}^{n-1} / m$, we have

$$
\frac{s_{0}}{2} B \subset \Pi \bar{P} \subset \frac{3 t_{0}}{2} B .
$$

Following exactly the proof of Lemma 4.2 from (16) on, with $K, L, r$, and $R$, replaced by $P_{k}, \bar{P}, r_{0}$, and $R_{0}$, respectively, yields

$$
r_{1} B \subset \bar{P} \subset R_{1} B
$$

where

$$
R_{1}=\frac{3 n \kappa_{n}}{\kappa_{n-1}}\left(\frac{3}{2}\right)^{1 /(n-1)} \frac{R_{0}^{n}}{r_{0}^{n-1}} \quad \text { and } \quad r_{1}=\frac{\kappa_{n-1} r_{0}^{n-1}}{2^{n} R_{1}^{n-2}} .
$$

Since $r_{1} \leq r_{0}$ and $R_{0} \leq R_{1}$, (38) also holds for $P_{k}$. By (6) with $a=1 /(n(n+4))$ again, we have

$$
\delta\left(P_{k}, \bar{P}\right) \leq c\left(n, r_{1}, R_{1}\right)\left(\frac{m \varepsilon_{0}}{2}\right)^{1 /(n(n+4))} .
$$

Consequently, we can guarantee that $\delta\left(P_{k}, \bar{P}\right)<\varepsilon / 2$ by choosing

$$
\varepsilon_{0}<\min \left\{\frac{\kappa_{n-1} r_{0}^{n-1}}{m}, \frac{2}{m}\left(2 \varepsilon^{-1} c\left(n, r_{1}, R_{1}\right)\right)^{-n(n+4)}\right\} .
$$

By (7), (15), and (39), $\varepsilon_{0}^{-1}$ can be chosen so that it is bounded by a polynomial in $r^{-1}$, $R$, and $\varepsilon^{-1}$. 


\section{Implementation}

With the assistance of a Western Washington University student, Chris Street, we have implemented Algorithms MinkData, BrightLSQ, BrightLP, and BrightNFacets. The various optimization problems, (LLS), (LP), and (NLS) in Phase I of Algorithms BrightLSQ, BrightLP, and BrightNFacets, are solved by using the optimization toolbox from MATLAB. The function fmincon from this toolbox, which employs a variety of nonlinear optimization techniques such as line search or Newton's method, is suitable for (NLS). Another function, Isqnonneg, is suitable for (LLS), and the function 1 inprog is used for (LP).

Note that if the simplex algorithm is used to solve (LP), then the number $l^{\prime}$ in Phase II of Algorithm BrightLP can be no greater than the number $k$ of constraints in (21). Then the number $m=2 l^{\prime}$ of facets of the output polyhedron is at most $2 k$. However, linear programming software, including MATLAB's linprog, often employs interior-point and other methods, and then the output may have more than $2 k$ facets.

Phase II of Algorithms BrightLSQ, BrightLP, and BrightNFacets calls Algorithm MinkData. When $n=2$, this is straightforward and can be done within MATLAB. When $n \geq 3$, our implementation of Step 1 of Algorithm MinkData again uses MATLAB's fmincon for solving the problem (NL). At each step of (NL), the computation of the volume $V(P(h))$ is done by a free $\mathrm{C}++$ program called Vinci obtainable from ftp: //ftp.ifor.math. ethz.ch/pub/volume/Volumen.html.

The conversion from $\mathcal{H}$-representation to $\mathcal{V}$-representation and computation of the convex hull in Step 2 of Algorithm MinkData are handled by the free qhull program available from http: //www. geom. umn. edu/software/downl oad/ qhull. html.

When $n=3$, qhull can provide the output in the form of a Mathematica graphics object which can be displayed by the Mathematica graphics package.

\section{Sample Reconstructions}

Figure 1 shows reconstructions of the regular octahedron with vertices at $( \pm 1,0,0)$, $(0, \pm 1,0)$, and $(0,0, \pm 1)$ from a finite set of values of its brightness function. The input consisted of exact brightness function measurements in the 21 directions corresponding to the spherical polar angles

$$
\left\{\left(\frac{2 r \pi}{5}, \frac{s \pi}{8}\right): r=0, \ldots, 4, s=0, \ldots, 4\right\} .
$$

Algorithm BrightLSQ was used to produce the convex polyhedron with 20 facets on the left of Fig. 1. The output of Algorithm BrightLP was the polyhedron with 28 facets on the right. In both cases the reconstructed polyhedron has the same brightness function as the octahedron in the above 21 directions, to 10 decimal places. The reconstructions took less than 3 minutes on a standard PC with a $466 \mathrm{MHz}$ Celeron processor and 128 MB RAM. (All but a few seconds of this time was required for Phase II.)

On the left of Fig. 2, a polyhedron $P$ is depicted that has 18 facets; it is the convex hull of the points $\left(0,0, \pm \frac{3}{2}\right), H$, and $-H$, where $H$ is the hexagon with vertices 

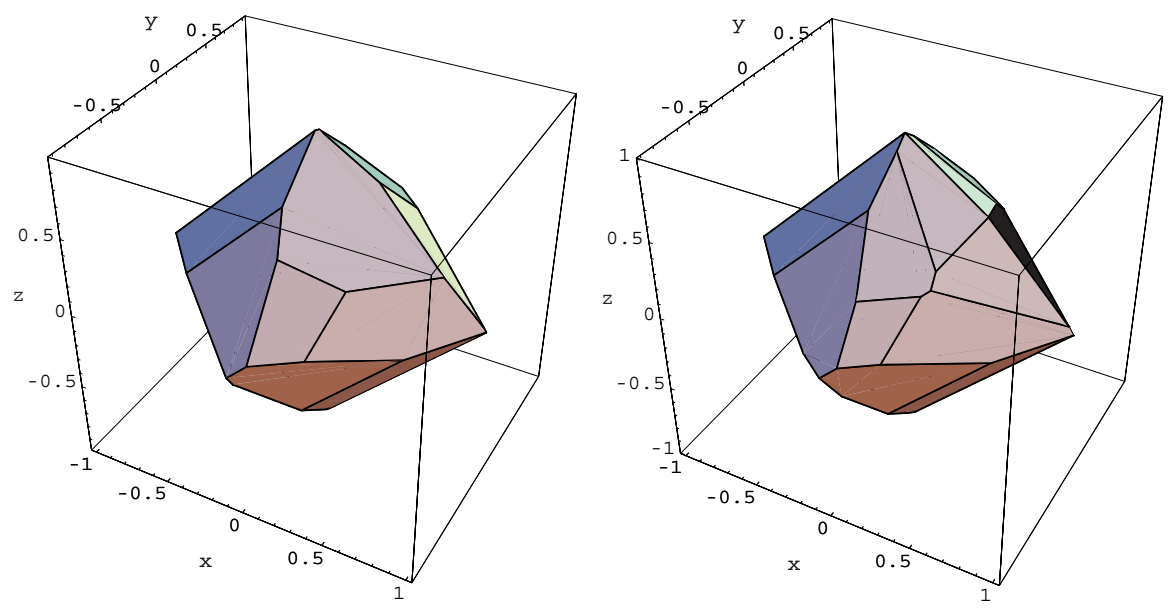

Fig. 1. Reconstructions of an octahedron from its brightness function.

$\left(\cos (k \pi / 6), \sin (k \pi / 6), \frac{1}{2}\right), k=1,3,5,7,9,11$. Reconstructions of $P$ using Algorithms BrightLSQ and BrightLP are the polyhedrons with 36 and 46 facets shown in the center and on the right of Fig. 2, respectively. The input consisted of exact brightness function measurements in the 36 directions corresponding to the spherical polar angles

$$
\left\{\left(\frac{2 r \pi}{7}, \frac{s \pi}{12}\right): r=0, \ldots, 6, s=0, \ldots, 6\right\} .
$$

In both cases the corresponding brightness values of the reconstructed polyhedron are within $2 \%$ of those of $P$. Times for reconstructions on the PC described above were
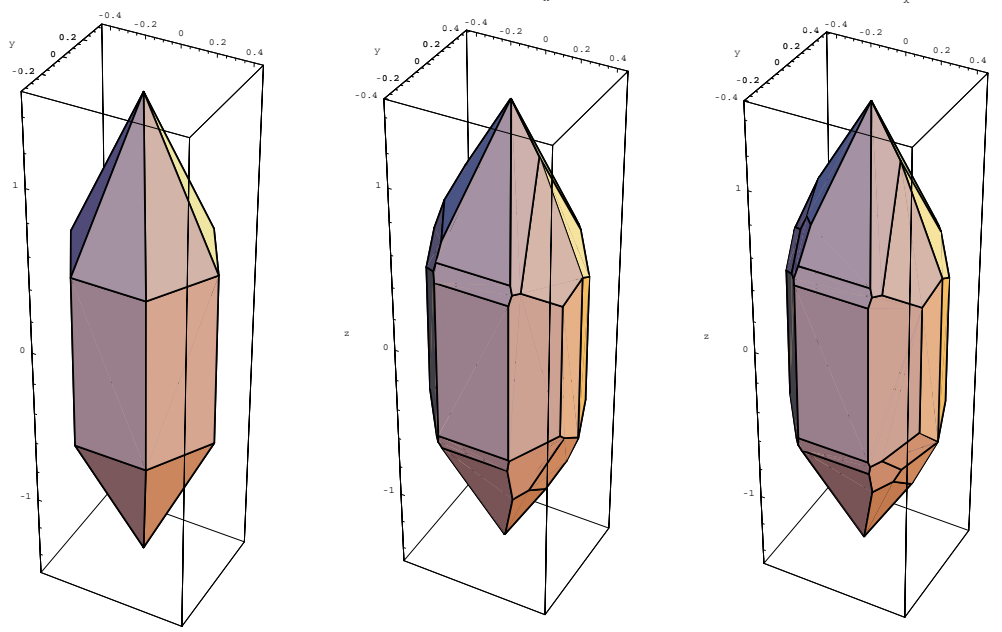

Fig. 2. Reconstructions of a polyhedron from its brightness function. 


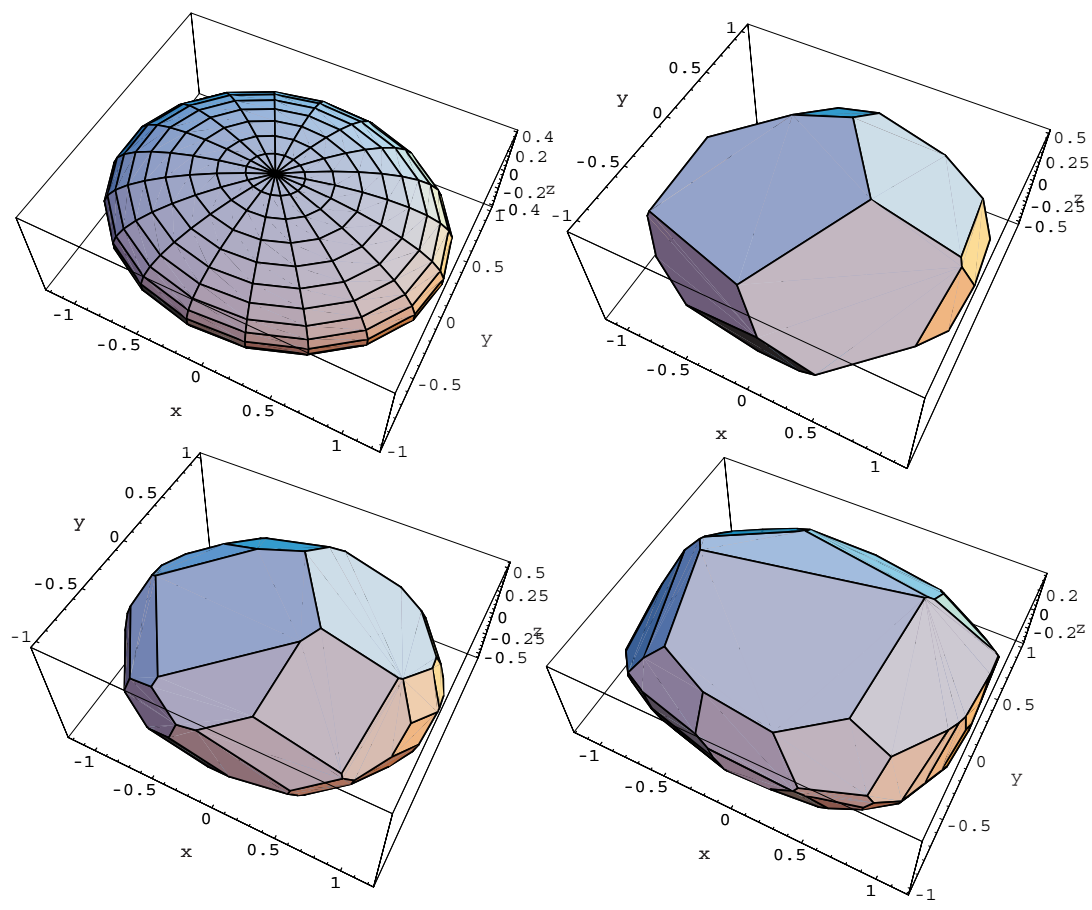

Fig. 3. Reconstructions of an ellipsoid from its brightness function.

as follows. Algorithm BrightLSQ required 2.5 minutes for Phase I and 6 minutes for Phase II, while Algorithm BrightLP took about 2 minutes for Phase I and 10 minutes for Phase II.

Figure 3 (top left) displays the standard ellipsoid

$$
\left\{(x, y, x): \frac{x^{2}}{a^{2}}+\frac{y^{2}}{b^{2}}+\frac{z^{2}}{c^{2}} \leq 1\right\}
$$

with parameters $a=1.2, b=1$, and $c=0.4$. At the top right and bottom left of Fig. 3 are reconstructions produced by Algorithms BrightLSQ and BrightLP, respectively. The input comprised a small data set of exact brightness function measurements of this ellipsoid in a certain set of 10 directions. This set of directions minimizes the spread $\Delta_{10}$ of the measurement directions in (26), and is congruent to that on the web page of $\mathrm{N}$. Sloane, in the library of best-known chordal packings of lines at http://www. research.att.com/ njas/grass/dim3/. Both the 20-faceted polyhedron at the top right of Fig. 3 and the 50-faceted polyhedron at the bottom left have brightness functions that agree up to 12 decimal places with that of the ellipsoid in this set of 10 directions. The reconstructions took about 2 minutes and 11 minutes, respectively, on the PC described above. (All but a few seconds of this time was required for Phase II.) 
As a more ambitious reconstruction, we have included at the bottom right of Fig. 3 a 72-faceted reconstruction of this ellipsoid, produced by Algorithm BrightLSQ from a larger data set of exact brightness function measurements taken in a set of 50 randomly generated directions. The corresponding 50 brightness function values of the reconstructed polyhedron agree with those of the ellipsoid to within $2 \%$. This reconstruction took about 3 minutes for Phase I and 23 minutes for Phase II.

\section{Concluding Remarks and Future Work}

It appears that our implementation of Algorithms BrightLSQ and BrightLP are quite successful. As was mentioned in the Introduction, these work with some noise present, and we plan to analyze the effect of noise in a future paper.

It is interesting that although Algorithm BrightLP should be considerably faster than Algorithm BrightLSQ, this was not generally true in our experiments. As expected, running times for Phase I of Algorithm BrightLP are somewhat shorter than those for Algorithm BrightLSQ, though the difference does not seem to be a dramatic one. However, the software we use seems to result in the number of facets in the output of Algorithm BrightLP being larger than that of Algorithm BrightLSQ, with a corresponding longer process time for Phase II. Since the time taken for Phase II is the dominant factor, our implementation of Algorithm BrightLSQ generally runs faster than Algorithm BrightLP.

Though we have had some success with Algorithm BrightNFacets in two dimensions, at present our implementation of it runs too slowly in three dimensions for practical use. Evidently this is due to the essential nonlinearity in (NLS) caused by the constraint (25). Nevertheless, this least squares problem is of a special type known as separable. In view of this, there is the possibility of improving performance by a variant of the Newton method, where each of the unknowns $\alpha$ and $\omega$ is treated separately in each iteration. See, for example, the article by Golub and Pereyra [10]. There is also the possibility of using the input brightness function values to improve on an arbitrary initial guess. In Section 9.3 of [8], various methods are discussed for estimating quantities such as surface area or volume from the brightness function, and the initial guess could be chosen accordingly.

In the future we plan to implement a completely different algorithm for reconstructing polyhedra with a prescribed number of facets. Gil Kalai observed that it should be possible to run Algorithm BrightLSQ or BrightLP and then perform a cluster analysis on the resulting output. Initial experiments indicate that this might be quite successful.

There are circumstances other than origin symmetry under which a convex body is determined, up to translation and reflection in the origin, by its brightness function. In order to understand this, let $K$ and $L$ be convex polytopes with $b_{K}=b_{L}$. Then the Blaschke bodies $\nabla K$ and $\nabla L$ satisfy $b_{\nabla K}=b_{\nabla L}$, so Aleksandrov's projection theorem implies that $\nabla K=\nabla L$. From the definition of the Blaschke body, we see that every facet of $K$ (or $L$ ) must be parallel to some facet of $\nabla K$ (or $\nabla L$, respectively), and hence that each facet of $K$ is parallel to some facet of $L$. It also follows that for each direction $u$, the sum of the volumes of the facets of $K$ orthogonal to $u$ must equal the sum of 
the volumes of the facets of $L$ orthogonal to $u$. Even if $K$ is "generic" in that it has no two facets parallel, and $K$ and $L$ have the same number of facets, one cannot in general conclude that $K= \pm L$, up to translation; for example, Figure 3.8 of [8] displays two noncongruent generic 9 -gons with equal brightness functions. However, these additional assumptions imply that if $K$ has facets of volumes $a_{j}$ and mutually nonparallel outer normals $v_{j}, j=1, \ldots, m$, then $L$ has facets of volumes $a_{j}$ and outer normals $\pm v_{j}$, $j=1, \ldots, m$. Minkowski's existence theorem then shows that if $K \neq \pm L$, there is a proper subset $I$ of $\{1, \ldots, m\}$ such that

$$
\begin{aligned}
\sum_{j=1}^{m} a_{j} v_{j} & =\sum\left\{a_{j} v_{j}: j \in I\right\}+\sum\left\{a_{j}\left(-v_{j}\right): j \in\{1, \ldots, m\} \backslash I\right\} \\
& =0
\end{aligned}
$$

so that

$$
\sum\left\{a_{j} v_{j}: j \in I\right\}=\sum\left\{a_{j} v_{j}: j \in\{1, \ldots, m\} \backslash I\right\}=0 .
$$

By (40) and Minkowski's existence theorem, there are (possibly degenerate) convex polytopes $K_{1}$ and $K_{2}$ such that $K_{1}$ has facets of volumes $a_{j}$ and outer normals $v_{j}$ for $j \in I$, while $K_{2}$ has facets of volumes $a_{j}$ and outer normals $v_{j}$ for $j \in\{1, \ldots, m\} \backslash I$. This is clearly impossible if $m \leq 5$, for example, so generic pentagons are determined, up to translation and reflection in the origin, among all pentagons by their brightness function.

In fact, it is quite straightforward to modify Algorithm BrightNFacets so that the origin symmetry is relaxed in both input and output. To do this, one replaces $N / 2$ by an arbitrary natural number $q \geq n$ and adds (8), with $m$ replaced by $q$, to the constraints to ensure a convex output. If required, a suitable initial guess $\left(\alpha^{(0)}, \omega^{(0)}\right)$ can be obtained as follows. Generate random unit vectors $\omega_{j}^{(0)}, j=1, \ldots, q$, and, for some fixed $\varepsilon_{0}>0$, random reals $\varepsilon_{0} \leq \alpha_{j}^{(0)} \leq 1, j=1, \ldots, q-n$. Then use (8), with $m$ replaced by $q$, to determine suitable $\alpha_{j}^{(0)}, j=q-n+1, \ldots, q$. Repeat the process until these $n$ numbers also lie between $\varepsilon_{0}$ and 1 . The formulation of convergence theorems for this algorithm in situations such as those described in the previous paragraph should be possible, but we do not pursue this here.

\section{Acknowledgments}

We have implemented most of the algorithms described in this paper. In connection with this, we thank Chris Street, a Western Washington University undergraduate student, for her expert programming assistance. Jim Little and Hamid Zouaki kindly sent data for testing our implementation of Phase II. We are obliged to Komei Fukuda for valuable information concerning computational geometry software and to Peter Gritzmann for his comments. Finally, we are grateful to Helmut Groemer and Markus Kiderlen for supplying some arguments used in Lemmas 4.1 and 4.2, and to the referees for perceptive comments that led to some significant improvements. 


\section{References}

1. C. B. Barber, D. P. Dobkin, and H. Huhdanpaa, The quickhull algorithm for convex hulls, ACM Trans. Math. Software 22 (1996), 469-483.

2. J. Bourgain and J. Lindenstrauss, Projection bodies, in: Geometric Aspects of Functional Analysis (1986/7), Lecture Notes in Mathematics 1317, Springer-Verlag, Berlin, 1988, pp. 250-270.

3. B. Büeler, A. Enge, and K. Fukuda, Exact volume computation for polytopes: a practical study, in: Polytopes-Combinatorics and Computation (Oberwolfach, 1997), Birkhäuser, Basel, 2000, pp. 131-154.

4. S. Campi, Recovering a centred convex body from the areas of its shadows: a stability estimate, Ann. Mat. Pura Appl. (4) 151 (1988), 289-302.

5. S. Campi, A. Colesanti, and P. Gronchi, Convex bodies with extremal volumes having prescribed brightness in finitely many directions, Geom. Dedicata 57 (1995), 121-133.

6. S. Campi, D, Haas, and W. Weil, Approximation of zonoids by zonotopes in fixed directions, Discrete Comput. Geom. 11 (1994), 419-431.

7. J. H. Conway, R. H. Hardin, and N. J. A. Sloane, Packing lines, planes, etc.: packings in Grassmannian spaces, Experiment. Math. 5 (1996), 139-159.

8. R. J. Gardner, Geometric Tomography, Cambridge University Press, New York, 1995.

9. R. J. Gardner and A. Volčič, Determination of convex bodies by their brightness functions, Mathematika 40 (1993), 161-168.

10. G. H. Golub and V. Pereyra, The differentiation of pseudo-inverses and nonlinear least squares problems whose variables separate, SIAM J. Numer. Anal. 10 (1973), 413-432.

11. P. Gritzmann and A. Hufnagel, On the algorithmic complexity of Minkowski's reconstruction problem, J. London Math. Soc. (2) 59 (1999), 1081-1100.

12. P. Gritzmann and V. Klee, On the complexity of some basic problems in computational geometry. I. Containment problems, Discrete Math. 13 (1994), 129-174.

13. P. Gritzmann and V. Klee, On the complexity of some basic problems in computational geometry. II. Volume and mixed volumes, in: Polytopes: Abstract, Convex and Computational (Scarborough, ON, 1993), Kluwer, Dordrecht, 1994, pp. 373-466.

14. H. Groemer, Geometric Applications of Fourier Series and Spherical Harmonics, Cambridge University Press, New York, 1996.

15. M. Grötschel, L. Lovász, and A. Schrijver, Geometric Algorithms and Combinatorial Optimization, Springer-Verlag, Berlin, 1988.

16. W. C. Karl, Reconstructing Objects from Projections, Ph.D. dissertation, Department of Electrical Engineering and Computer Science, Massachusetts Institute of Technology, Cambridge, MA, 1991.

17. M. Kiderlen, Non-parametric estimation of the directional distribution of stationary line and fibre processes, Adv. in Appl. Probab. 33 (2001), 6-24.

18. L. Lamberg, On the Minkowski problem and the lightcurve operator, Ann. Acad. Sci. Fenn. Ser. A I Math. Dissertationes 87 (1993).

19. J. B. Lasserre, An analytical expression and an algorithm for the volume of a convex polyhedron in $\mathbb{R}^{n}$, J. Optim. Theory Appl. 39 (1983), 363-377.

20. A. S. Lele, Convex Set Reconstruction from Support Line Measurements and Its Application to Laser Radar Data, M.Sc. dissertation, Department of Electrical Engineering and Computer Science, Massachusetts Institute of Technology, Cambridge, MA, 1991.

21. A. S. Lele, S. R. Kulkarni, and A. S. Willsky, Convex polygon estimation from support line measurements and applications to target reconstruction from laser radar data, J. Opt. Soc. Amer. A 9 (1992), 1693-1714.

22. J. Lemordant, P. D. Tao, and H. Zouaki, Modélisation et optimisation numérique pour la reconstruction d'un polyèdre à partir de son image gaussienne généralisée, RAIRO Modél. Math. Anal. Numér. 27 (1993), 349-374.

23. J. J. Little, An iterative method for reconstructing convex polyhedra from extended Gaussian images, in: Proc. AAAI, National Conf. Artificial Intelligence (Washington, D.C.), 1983, pp. 247-250.

24. C. A. Rogers, Covering a sphere with spheres, Mathematika 10 (1963), 157-164.

25. R. Schneider, Convex Bodies: The Brunn-Minkowski Theory, Cambridge University Press, Cambridge, 1993.

26. M. A. Sumbatyan and E. A. Troyan, Reconstruction of the shape of a convex defect from a scattered wave field in the ray approximation, J. Appl. Math. Mech. 56 (1992), 464-468.

Received November 15, 2001, and in revised form July 2, 2002. Online publication December 20, 2002. 